\title{
Féeries
}

Études sur le conte merveilleux, XVII ${ }^{-} \mathrm{XIX}{ }^{\mathrm{e}}$ siècle

\section{Politique du conte}

\section{Les leçons de lumières de $\mathrm{M}^{\text {lle }}$ de Lubert}

\section{Marie Vorilhon}

\section{OpenEdition}

Journals

Édition électronique

URL : http://journals.openedition.org/feeries/170

DOI : $10.4000 /$ feeries. 170

ISSN : 1957-7753

\section{Éditeur}

UGA Éditions/Université Grenoble Alpes

\section{Édition imprimée}

Date de publication : 1 février 2006

Pagination : 353-368

ISBN : 2-84310-082-8

ISSN : $1766-2842$

\section{Référence électronique}

Marie Vorilhon, "Les leçons de lumières de M"le de Lubert », Féeries [En ligne], 3 | 2006, mis en ligne le 07 février 2007, consulté le 08 septembre 2020. URL : http://journals.openedition.org/feeries/170 ;

DOI : https://doi.org/10.4000/feeries. 170

Ce document a été généré automatiquement le 8 septembre 2020

(c) Féeries 


\title{
Les leçons de lumières de $\mathrm{M}^{\text {lle }}$ de Lubert
}

\author{
Marie Vorilhon
}

On a fait ces derniers temps des contes de fées très ingénieux, où sous le voile de la construction,

l'on trouve quantité d'instructions utiles' ${ }^{1}$.

1 SI LES PERSONNAGES DES CONTES DE MLLE DE LUBERT participent d'une fantaisie commune et représentent les passions de tout lecteur, comme il est de bon ton dans les contes de fées, il s'agit avant tout de mettre en scène des êtres confrontés au pouvoir. En effet, rois et reines, mais surtout princes et princesses, suivent bon gré mal gré une démarche de découverte de la chose politique. Sous l'impulsion des fées, ils servent à l'auteur de témoins de la mise en place d'une politique éclairée, qui lutte contre les limites que l'absolutisme impose à l'homme social et à la pensée.

$2 \mathrm{Au} \mathrm{XVIII}^{\mathrm{e}}$ siècle, la prépotence d'un seul homme et d'une seule religion commence à être remise en question. La préoccupation politique est au centre des réflexions des Lumières et présente manifestement au fil des contes de Mlle de Lubert. Les contes de la fin du siècle précédent faisaient l'éloge du pouvoir. Ceux du XVIII ${ }^{\mathrm{e}}$ siècle semblent vouloir le déstabiliser.

3 Tecserion, le premier conte de Mlle de Lubert, est précédé dès sa parution, en 1737, d'un discours préliminaire intitulé : Il y a de la raison à s'amuser par la folie ${ }^{2}$. Voici définie une écriture dont l'objet s'avère on ne peut plus paradoxal. Cette préface, dont le but est de préparer le lecteur à son entrée dans le conte qui va suivre et dans l'œuvre de Mlle de Lubert, s'appuie effectivement sur un paradoxe: le lien proposé d'emblée entre « raison » et "folie ». Le conte de fées, genre rejeté, affublé du terme radical et très insuffisant de "sous-genre ", prendrait de fait une dimension toute particulière et qui mérite qu'on s'y attarde. C'est avec la plus grande audace que Mlle de Lubert donne à ces petits textes une profondeur philosophique. Si l'on considère le conte a priori, en suivant le cours de ce «Discours préliminaire ", il s'agit d'un écrit de peu de sens, qui flatte bien plus l'affect que l'intellect, plus proche semble-t-il, de la sensation que de la réflexion, plus proche du corps que de l'âme, et proche finalement de quelque chose qui 
fait peur et qu'on ne maîtrise pas : le fantasme. Ce petit genre surgit aux antipodes de toute construction raisonnante en matière de philosophie ou de politique.

L'auteur semble demander au lecteur de se libérer de toute contingence intellectuelle, de lire le texte avec un esprit innocent. C'est le vide, que procure cette agréable sensation, qui va permettre un appel d'air philosophique, pour surprendre une réflexion neuve. Car derrière l'apparente futilité se cache une réflexion toute politique, digne de l'esprit en mouvement du Siècle des lumières.

Mlle de Lubert en vient au fait, associant la petitesse du genre : « Tecserion est un conte de fées, on l'avoue humblement ${ }^{3}$ ", à son importance dialectique : "un philosophe qui ne comprend pas que des hommes raisonnables puissent s'occuper d'un conte de fées, n'est point philosophe ${ }^{4} »$. Ce genre, à première vue mineur, apparaît donc comme essentiel. En effet, si l'on considère le mot d'ordre que Mlle de Lubert avance, tant pour l'écriture que pour la lecture de ces bagatelles, il s'agit avant tout d'«amusement ". Cette démarche marque le caractère éphémère du texte féerique et en fait, de prime abord, un texte de l'impression. En effet, ces féeries, dont les personnages et les motifs nous semblent familiers, apparaissent comme des moments de distraction et d'évanescence, propres aux esprits oisifs et flottants. Elles sont la consécration de la lecture-instant. Cependant, si l'on considère le terme " amuser ", à la fin du XVII siècle, il prend un sens tout à fait significatif quant à la définition générique que tente d'effectuer Mlle de Lubert : "faire durer une chose sans arriver au fait, afin de faire perdre de vue le but de l'entreprise ${ }^{5} »$. La futilité devient ruse, et l'égarement nécessaire dans la fantaisie, qui procède à l'écriture comme à la lecture, s'avère être une sorte de pacte entre le lecteur et l'auteur. Plus qu'une détente, le conte devient un divertissement réflexif, un piège salutaire pour esprits disponibles.

Mlle de Lubert choisit une esthétique légère pour développer une éthique nouvelle et une réflexion politique dont la densité est là pour nous étonner. « Le divertissement est un bien, et l'ennui un mal ${ }^{6}$ ». L'ennui, c'est peut-être cette réflexion soumise à un ordre convenu, à une norme rigoureuse, qui veut que sous telle forme se trouve tel sens, et qui, pour son bonheur comme pour son malheur, confine le conte de fées au non-sens. Le divertissement, c'est l'intuition comme rejet de la norme, rejet des formes convenues et académiques, la liberté d'une pensée qui court au fil de l'imaginaire pour mieux impressionner l'esprit. L'éthique s'extirpe ainsi de la bagatelle esthétique, de la légèreté du conte, avec d'autant plus de facilité qu'on n'avait pas prévu qu'elle se trouvât là. Quelle est la lecture politique que proposent les contes de Mlle de Lubert, du Siècle des Lumières?

Le miroir d'une décadence

7 Derrière le ballet des individualités stéréotypées dans une outrance qui sert l'absurde, le conte de Mlle de Lubert propose des personnages en devenir politique. Elle épingle les stéréotypes mondains $\mathrm{du}$ xviII ${ }^{\mathrm{e}}$ siècle, les décortique avec délice et leur fait subir, in vitro, toutes sortes de métamorphoses et manipulations savantes, afin qu'ils se retranchent naturellement dans leurs limites. Sous sa plume, le conte devient un univers politique expérimental. Et si tout était autrement, que se passerait-il ? Le conte permet ce qui, dans un roman, serait censuré. On ne se méfie pas de la fantaisie, elle est trop légère, elle échappe.

8 Sexe fort, sexe faible, tout se mélange dans les contes de Mlle de Lubert; et ce qui pouvait apparaître comme un équilibre naturel s'avère fragile. En effet, le genre humain, en particulier son représentant masculin, n'est pas tout à fait à son avantage 
dans les contes de Mlle de Lubert. Souvent dépeints dans des situations ridicules, les rois ou les princes sont habituellement passifs et enclins à la mollesse. Les reines et les princesses (un peu plus agissantes) ne sont pas non plus sans avoir leurs défauts, et notamment celui de repousser l'homme dans cette apathie particulière. Ainsi le roi, dans ces récits féeriques, est un homme qui se repose sur un ordre établi, qui n'a pas la force d'en changer et qui se complaît dans une médiocrité coutumière et somme toute confortable. Dans La Princesse Sensible et le prince Typhon, l'auteur, en quelques lignes, trace le portrait moral du roi, père de Sensible :

Ce prince était le meilleur de tous les hommes, il n'exigeait de ses sujets aucune espèce de contrainte, sa principale maxime était de ne rien changer aux usages établis, et dans tous les cas où il fallait décider, il répondait toujours comme à l'ordinaire ${ }^{7}$.

Cependant cette passivité très forte des hommes - rois, pères, et bientôt dieu(x) -, est souvent l'effet d'une autorité féminine très forte. Ainsi dans La Princesse Lionnette et le prince Coquerico, si le prince prend les allures efféminées d'Achille à la cour de Lycomède, c'est qu'il se trouve étouffé par une mère qui refuse de faire de son fils un homme. L'éducation du prince Coquerico se trouve être l'énoncé de l'éducation idéale des jeunes filles aux $\mathrm{XVII}^{\mathrm{e}}$ et $\mathrm{XVIII} \mathrm{e}^{\mathrm{e}}$ siècles. Lecture, musique, peinture et travaux de couture, entraînent le roi en devenir vers l'oisiveté raffinée et la mollesse habituelle d'une aristocratie féminine.

Or au contraire du prince, l'auteur délègue à sa promise, la princesse Lionnette, des attributs d'une virilité non dissimulée. On la voit ainsi parée des attributs de Diane chasseresse, parcourant les forêts à dos de lionne. Le topos habituel du prince et de la princesse se trouve bouleversé. La princesse devient, chez Mlle de Lubert, l'élément viril du couple, elle est symbolisée par le lion, roi des animaux - dont le coq, son amant, devient sujet - et de la vie sauvage en général, monde sans limites, royaume infini. Le prince au contraire, apparaît doté de qualités féminines : la petitesse - celle du coq, par rapport à la taille du lion -, la peur des armes, les occupations minutieuses. Son règne est celui de la basse-cour, monde cloisonné de la vie domestique. Règne ridicule émaillé d'un vain orgueil.

11 Le ridicule d'un bon roi empêché, est contrebalancé, au fil des contes de Mlle de Lubert, par l'image du tyran burlesque. Plus la puissance d'un personnage est forte, plus son ridicule en est augmenté. Si l'on examine les différents portraits des tyrans qui sévissent dans les contes, on est étonné de la monstruosité burlesque de ces personnages. Tecserion, dans le conte du même nom, Pendagrugeon dans Blancherose ou encore Croquignolet dans La Princesse Coqued'œuf et le prince Bonbon, en sont des exemples probants. En voici les portraits successifs :

Imaginez-vous une petite figure, haute d'environ deux pieds, dont la moitié était en visage, mais si maigre et étroit, qu'on le perdait presque de vue dans un bonnet à oreilles doublé d'hermine. Une barbe aussi blanche et qui semblait être du même poil que la fourrure de son bonnet, lui descendait plus bas que le genouil. Une couronne de la hauteur d'un pied et d'un seul diamant brillant, faisait un effet ridicule sur sa tête; et sa robe de velours pourpre et noir, toute rattachée d'agrafes et de diamants, était à la vérité la plus belle chose du monde, mais en même temps, la plus inutile, pour rendre beau le roi des Autruches ${ }^{8}$.

Il était haut comme environ un grand géant; sa tête, portée sur un long col de dromadaire, avait la vraie physionomie d'un sanglier, excepté qu'il lui sortait des deux côtés du front deux espèces de tuyaux, au bout desquels étaient ses deux yeux ${ }^{9}$. 
Il était autrefois un roi qui avait le nez si long, si long, que quoique l'extrémité fût roulée sur une bobine et portée par deux pages qui n'étaient point payés et qui s'entretenaient à leurs dépens, la partie cartilagineuse du nez était encore si vaste et si peu flexible, qu'on avait été obligé d'abattre tous les coins des rues de la capitale, pour donner au prince la facilité de tourner lorsqu'il allait à la promenade. Or comme ce nez, qui croissait toujours, était sujet à d'importunes démangeaisons, les médecins ne trouvèrent d'autres remèdes pour les apaiser, que de faire donner sans cesse des croquignoles au bon prince, ce qui le fit surnommer le roi Croquignolet ${ }^{10}$.

12 De ces trois tyrans, personnages de la démesure en position omnipotente, Tecserion se voit réduit au minuscule, alors que les deux autres sont affublés de caractères gigantesques. Chacun de ces rois est doté d'un organe exubérant: la barbe de Tecserion, qui pourrait symboliser la sagesse du vieillard, est employée à rebours, car elle est immédiatement ridiculisée par celui qui la porte; les yeux de Pendagrugeon mettent en avant son pouvoir inquisiteur et pervers. Quant au nez de Croquignolet, c'est l'image de sa volonté de puissance - sexuelle puis politique -, réduite à néant par l'encombrement que cet organe lui procure.

13 Chacun de ces personnages donne une impression d'amas, de flou, de grouillement par son esthétique même: Tecserion, composé de taches blanches, pourpres et noires, porte les couleurs $\mathrm{du}$ pouvoir de manière diluée et grotesque. Même son nom, labyrinthique, égare le lecteur: "Tecserion » est l'anagramme de "sec et noir ». Ces deux adjectifs peuvent désigner à la fois un caractère physique et un trait moral. Il peut être «sec » autant par sa maigreur, qui en fait un personnage en voie de disparaître, que par la rigidité de son pouvoir ; et « noir », par l'état de ses humeurs, mais aussi par la couleur de sa peau. Ce personnage minuscule et sombre prend en effet des allures de pygmée. La description de Pendagrugeon est aussi fantasmatique: ogre hybride, chimère ridicule, produit du mélange entre le dromadaire, le sanglier et l'escargot: l'exotique, le sauvage, et l'ambiguïté sexuelle mis bout à bout. C'en est trop pour un seul monstre! Quant à Croquignolet, il ne va pas sans un abondant essaim de courtisans qui l'entourent pour lui donner force croquignoles. Rappelons que les croquignoles sont des chiquenaudes, des nasardes, exercice habituellement pratiqué par les écoliers et destiné aux moqueries. Par ce portrait, l'auteur singe les aspects de la cour, l'infantilise - ainsi que son roi - et la réduit à une ruche active à des sornettes, fruits de la personnalité masochiste d'un souverain ridicule.

Lorsqu'elle parle du microcosme de la cour, Mlle de Lubert ne mâche pas ses mots. On décèle en effet sous de nombreux traits, une critique acerbe de la mondanité, un état néfaste que Phila et Mulidor ont choisi de quitter. Ce passage, situé en incipit de La Princesse Lionnette et le prince Coquerico, offre sans détours le fil rouge de la lecture du conte :

Dans les montagnes de la Circassie, il y avait un vieillard avec sa femme qui s'étaient retirés du monde ; las d'en avoir essuyé les caprices, ils s'étaient fait une retraite commode d'une caverne, qui s'étendait assez loin sous un des monts, et leur solitude n'était troublée que par la crainte de se voir mourir. Ils avaient vécu dans les cours, ils en connaissaient tout le faux; et loin de regretter les places brillantes qu'ils avaient occupées, ils plaignaient ceux que l'ambition ou le peu d'expérience rendaient susceptible de les désirer ${ }^{11}$.

Dans la simplicité que dicte une vie retirée, avec la vieillesse leur est venue la sagesse, cependant que l'avancée dans l'âge marque la péremption d'un courtisan. Mlle de Lubert fait dans ce conte l'éloge d'une vie simple génératrice d'une pensée vraie. La 
caverne, sombre et platonicienne, étape nécessaire à une amorce de la Connaissance, s'oppose au faux brillant de la cour dans lequel sont projetés les plus jeunes recrues, petits maîtres à la mode et autres favorites d'un jour. Dans ce retour aux choses simples, le désir redevient univoque, et l'Idée peut être révélée, alors que dans le monde centripète de la cour, le désir est ubiquité, il se travestit et se trompe en voulant tromper.

critique de la cour est récurrente dans les contes de Mlle de Lubert, et devient le lieu de satires plus serrées. Dans La Princesse Sensible et le prince Typhon, par exemple, on sent que s'opère un glissement de la cour au salon, microcosme qui apparaît aussi mortifère que la cour, car il adopte lui aussi un système féodal - appliqué à la pensée, ici, puisque, pour l'heure, lieu d'éducation de la princesse. La cour de Sensible est dirigée par la fée Prudalie, envoyée par la fée Capable pour faire de la princesse une jeune personne policée. L'effet néfaste s'en fait ressentir immédiatement, produisant une princesse atrophiée - "à l'âge de quinze ans, elle n'avait tout au plus que trois pieds ${ }^{12}$ » craignant les changements d'air, et passant le plus clair de son temps sous « de grandes cloches de verre ${ }^{13}$ ", apprenant "l'art de parler par les doigts ${ }^{14}$ » afin de ménager sa poitrine, préférant la nuit au jour, moins propice à l'imagination amoureuse dans laquelle elle se complaît. La voilà donc sans cesse " le dos tourné contre les lumières ${ }^{15}$ ».

La préciosité, éducation artistique qui, poussée à l'outrance, bride le naturel au profit de l'art obligatoire, a comme salon le plus en vue "l'Hôtel de Rambouillet ${ }^{16}$ ». Clairement dénoncé dans La Princesse Sensible et le prince Typhon, ce raffinement extravagant de la pensée est, selon l'auteur, une des meilleures manières de la perdre.

Dans Cornichon et Toupette, au-delà de la cour et d'une artificielle préciosité, c'est l'organisation sociale dans son ensemble, qui est dénoncée. La fée de l'île, Dindonnette, très à l'écoute des désirs des hommes, a cru bon d'enchanter la seule source de l'île, afin de rajeunir les plus vieux, et de vieillir les plus jeunes. Par l'adoption d'aspects rococo, dans le mélange, qu'il rend labyrinthique, des générations, le conte fait montre d'une abondance formelle galopante, qui se joue de l'âme. L'aspect nie le sens qu'il transporte d'habitude. Et toujours le ton est léger ou désinvolte.

Dans la scène qui suit, l'auteur démontre, par l'absurde, la nécessité d'une instruction politique. Cette nécessaire réflexion politique, leitmotiv des contes de Mlle de Lubert, apparaît fréquemment sous l'égide de l'exubérance. Brouiller les pistes lui permet, paradoxalement, d'exposer plus clairement sa pensée. En entraînant les formes vers l'exagération, l'auteur révèle un fond raisonnable - et raisonnant -, une morale à accepter sous peine de chaos. Car le désordre qui suit est dû notamment à la confusion des âges et des expériences, en politique comme en toutes choses :

Celui qui tombait dans l'enfance au sortir de la vieillesse, n'emportait aucun souvenir de ses connaissances passées. Un monde nouveau s'offrait à sa vue étonnée, et l'apprentissage qu'il en fallait faire pour se rendre utile à soi-même et aux autres, était toujours prévenu par le terme fatal où la décrépitude diminue l'exercice de la raison, en même temps qu'elle suspend ou interdit l'usage des facultés corporelles. De là, la privation entière de toute instruction, qui entraîne celle de l'idée du bien général, et des moyens d'y trouver le sien propre ${ }^{17}$.

Les relations sociales sont, de fait, faussées par le manque d'expérience et par l'impossible transmission de cette expérience. La politique, littéralement «art de la cité », est tronquée par cette incapacité à unir les savoirs pour les appliquer, dans une temporalité qui ne prend plus en compte l'expérience sociale. Le temps, la notion 
même d'Histoire échappe à l'entendement de ces humains exaucés dans leur folie. Le désir de régner sur le temps fait perdre le sens de l'Histoire, et la vie politique s'avère impossible, entrainant par là même la chute de l'homme. Folies humaines et folies de l'art se confondent, pour revendiquer de manière plus explicite les démarches rationnelles de réflexions politiques vers lesquelles nous entraîne le texte.

L'éducation politique

21 Critique amère de la cour et de la société mondaine comme microcosme étouffant, le conte prône tout d'abord un retour à l'éden originel, à travers l'idéal pastoral que l'on trouvait déjà dans certains romans du XVII ${ }^{e}$ siècle, et que Mlle de Lubert n'a pas manqué de lire. Dans cet éden, l'enfant va pouvoir devenir un homme et le prince, un roi, pour s'en retourner instruit et sage, l'un vers sa maîtresse, qui finit par supporter sa présence, et l'autre auprès de ses sujets.

L'organisation sociale remise en question, par l'imagerie rococo du mélange des corps et des âges, Mlle de Lubert propose quelques princes aptes à remettre un peu d'ordre en politique. Le "prince ", qui signifie littéralement " premier », doit être l'exemple, et doit donner l'impulsion pour une réalisation sociale. Plus que toute chose, derrière cet homme se découvre une nouvelle politique, se dessine l'envie d'un renouveau social.

Le prince Bonbon, héros de La Princesse Coqued'œuf et du prince Bonbon, figure une sorte de Candide avant la lettre. Son nom peut être entendu comme une répétition de l'adjectif «bon", synonyme de naïf, d'ingénu. Avec ce nom proche du pléonasme, Bonbon a tout d'un esprit neuf, simple, injustement meurtri lorsqu'il est chassé de son royaume. Chassé de sa patrie, "pays du père ", par une marâtre despotique, encore une fois. Le voyage qu'il entreprend est picaresque par bien des aspects. C'est un voyage initiatique, fait d'accumulations de rencontres toutes plus étonnantes les unes que les autres, et qui contribuent à former le héros politique. Cependant Bonbon apparaît comme un être oisif, dépité, qui ne vit que d'artifices, ceci pour combler le manque initial : la tyrannie matriarcale qui sévit chez lui.

24 Ce prince à croquer, aux allures superficielles, aux parures exubérantes de sucreries et aux mœurs apparemment dissolues, ne goûte pas sa vie de banni comme il le devrait. Il se réserve la liberté de choisir une politique, après cette période d'errance, et de surmonter ses troubles passés. Ce long voyage, hors de son royaume, lui sert finalement de retraite, et représente pour lui une mort sociale salutaire qui va marquer le début d'une réaction et l'amorce d'une nouvelle réflexion politique.

Le temps du conte de fées élogieux vis-à-vis du pouvoir est révolu. Les princesses ne se préparent plus au mariage - donc à l'accession au pouvoir - dans les sous-sols de châteaux sans fenêtres, dans des appartements tapissés des exploits du bon Roi Soleil ${ }^{18}$. Bien au contraire, les princesses de Mlle de Lubert prennent des leçons de géographie ${ }^{19}$, étudient les politiques étrangères, s'ouvrent au monde. Une nouvelle attitude s'annonce, plus contestataire.

L'homme cultivé du XvIII ${ }^{e}$ siècle observe le monde, les individus qui le composent, et se laisse étonner au sens philosophique du terme. Il se laisse surprendre par la différence, par la multitude des impressions qu'il intègre, et retranscrit toutes les réflexions que lui inspire cette diversité. La prose du $\mathrm{XVIII}^{\mathrm{e}}$ siècle se rapproche de la vérité des individus. Le roman se développe, genre de la vérité, mais aussi genre dont la forme ouverte permet une plus grande liberté. Et pour le conte, toute féerie mise à part, il en est de même. 
27 S'il est alors une attitude qui permet l'étonnement philosophique et déclenche une réflexion politique, c'est celle commune au Siècle des lumières, de ce qu'on appelle le Grand Tour. Aux XVII et XVIII ${ }^{e}$ siècles, les jeunes aristocrates anglais avaient coutume de faire un voyage de formation à la fin de leurs études. Ce voyage leur permettait de parfaire leur connaissance des langues et des gouvernements des régions visitées. On retrouve aussi dans les familles nobles françaises cette pratique du Grand Tour. Certains accusent ce procédé comme une menace de l'intégrité de la jeunesse; les philosophes des Lumières, au contraire, l'encouragent et défendent volontiers l'utilité de cette démarche initiatique. Au fil du siècle, le Grand Tour devient moins formaliste, et les voyages ainsi effectués sont plus souvent motivés par la curiosité de découvrir les sciences, les arts et les techniques, et plus ouverts aux aspects ludiques du dépaysement.

Dans les contes de Mlle de Lubert, la notion de Grand Tour comme découverte politique est très présente. De manière assez systématique dans les contes de fées, le héros effectue une quête, souvent matérialisée par un voyage. Mais Mlle de Lubert ne confine pas cette étape à une démarche intérieure, elle encourage aussi les personnages à parfaire leur santé intellectuelle, ou donne clairement un sens politique à la confrontation du prince avec l'étendue de l'univers.

Ainsi, dans Cornichon et Toupette, deux attitudes s'opposent : celle des habitants de l'île de la Fontaine, qui pensent que l'eau qui les entoure marque les limites du monde, à l'image des penseurs rétrogrades du siècle, dont la pensée concourt à l'étouffement intellectuel ; et celle, tournée vers une respiration cosmopolite, de la fée Selnozoura, «qui, par l'avis des médecins faisait ordinairement deux fois la semaine le tour de la terre pour changer d'air, et pour trouver quelque soulagement à des inquiétudes dans les jambes qui la tourmentaient ${ }^{20}$ ». La lourdeur que ressent la fée dans les jambes représente symboliquement une certaine lourdeur intellectuelle; et la prescription médicale, la recherche d'une amélioration par l'exploration de ce qui est inconnu. Cette ouverture à d'autres horizons, d'autres réactions, d'autres "airs", consacre la démarche de découverte des cultures étrangères, pour l'entraînement à une réflexion systématique sur sa propre culture.

Dans La Princesse Lionnette et le prince Coquerico, l'idée de Grand Tour comme nécessaire à cette nouvelle philosophie, est développée de manière originale : le prince est enlevé par une fée qui va tenter de faire de lui un homme, mais aussi un politique. Pour cela, elle l'enferme dans un palais qui dispose d'une «Galerie des Plans ${ }^{21}$ » ou «Galerie de l'Univers ${ }^{22} »$, permettant de considérer d'un coup d'œil l'ensemble du monde, et les actions des rois et des sujets, évoluant en miniature sur une immense table qui représente le monde en action.

31 Des idées nouvelles apparaissent dans les contes de Mlle de Lubert, fidèles à l'esprit des Lumières. Elle cite volontiers Montesquieu ${ }^{23}$, le plagie parfois, pour en adopter les attitudes ou les réflexions. De manière détournée, mais non moins efficace, par le truchement du conte, Mlle de Lubert expérimente in situ plusieurs théories politiques alors à l'étude.

Dans La Princesse Sensible et le prince Typhon, la compréhension politique, la genèse du pouvoir, se fait tout d'abord par l'appréhension de soi et de la relativité de toute chose. Par expérience, le prince Typhon, projeté par la fée Capable dans le Temple de l'amourpropre, apprend à tenir compte des différentes humeurs, des différentes perceptions 
des personnes selon leur condition. L'appréhension de la relativité des choses induit l'éloge de la tempérance et de la nécessaire tolérance.

Dans Tecserion, où il est beaucoup question de politique, l'auteur nous entraîne, sous couvert d'interlude, dans une Forêt magique qui vient de subir l'intrusion d'un étranger: un Tilleul vient en effet de s'installer dans la Forêt des Chênes, ce qui provoque la convocation du grand Conseil par le Chêne protecteur :

Il est né ce matin un Tilleul dans notre empire, et c'est pour vous en avertir que je vous rassemble ici ce soir : mais devons-nous le souffrir parmi nous? [...] Il est sûr encore qu'il est étranger, et qu'il a passé ici d'un autre État, mais aussi cette même qualité d'étranger, semble nous défendre d'user de violence contre un malheureux qui nous demande un asile; d'ailleurs il est beau et promet un bel ombrage : nous rendrons-nous à cette apparence qui est souvent trompeuse ? Ou bien suivronsnous les lois de l'empire qui n'est fondé que sur ces mêmes lois qui défendent expressément de souffrir parmi nous aucun arbre d'une espèce différente de la nôtre ? L'affaire est embarrassante et a besoin de vos lumières. [...]

Ah que proposez-vous? interrompit un jeune Chêne. Faut-il prévenir par des voies cruelles et injustes, des maux qui peut-être n'arriveront point ? [...] Après tout, la force de notre État peut-elle être affaiblie par les étrangers qui peuvent s'y établir? N'est-ce pas une marque de notre puissance que de pouvoir leur donner un asile ${ }^{24}$

Cette réflexion sur la notion d'étranger prend place sous les yeux d'une princesse étonnée, qui écoute attentivement la leçon. L'auteur déroule devant ses yeux - et les nôtres - les arguments pro ou contra, proposant une diversité d'intervenants et d'opinions. Cependant la préférence est de mise pour les paroles du jeune Chêne, dont la réflexion s'appuie sur des idées nouvelles. Il est le représentant de cette jeune génération de penseurs qui commencent à peser fortement sur les excès du pouvoir. On peut encore une fois percevoir l'influence des Lettres persanes de Montesquieu, publiées une quinzaine d'années avant Tecserion: la richesse que peut apporter un étranger au pays qui l'accueille va dans le sens du cosmopolitisme, de l'envie de découvrir et d'étudier d'autres cultures, d'autres formes de politique, pour en conserver le meilleur, car l'étranger va permettre de diversifier les points de vue, et d'enrichir deux cultures : la sienne et celle du pays qui l'accueille.

L'idée de l'interpénétration des influences et des points de vue se retrouve dans Cornichon et Toupette, lorsque Selnozoura, ne pouvant plus se déplacer pour son tour de terre quotidien, envoie des sylphes recueillir les airs des pays qu'elle aurait dû survoler. Cependant, une fuite dans les outres provoque le mélange intempestif des airs récoltés. L'effet est surprenant :

[...] ce jour fut marqué par des actions si pleines de sagesse, de la part de la fée, et si éloignées des extrêmes où l'usage de l'air unique de certains pays l'avait portée quelquefois, qu'elle s'en aperçut elle-même, et demanda du même air pour le lendemain. Alors les Sylphes ne firent pas de difficultés d'avouer leur aventure. Elle servit à éclairer la fée, et lui fit connaître qu'il y a du bon partout. Que les excès même se tempèrent les uns par les autres, et qu'enfin du concours de mille qualités les plus opposées, il résulte une qualité moyenne qui est la bonne. Fixée à cette opinion, elle ne voulut plus user que d'un air composé. Et c'est ce qu'on appela le bon air $^{25}$.

Symboliquement, la fée Selnozoura a subi les influences mélangées de plusieurs cultures, et son despotisme a été éclairé par des avis différents. De fait, la fée gouverne de manière plus sage et réfléchie. On ne peut manquer de percevoir dans cette anecdote féerique la nécessité de séparation des pouvoirs, une des idées que Montesquieu développe dans L'Esprit des lois, publié pour sa totalité en 1751, c'est-à-dire 
un an avant la parution de Cornichon et Toupette. En effet, pour pacifier les relations sociales, Montesquieu propose une répartition du pouvoir en " corps intermédiaires »: le corps législatif, le corps judiciaire et le corps exécutif, ceci afin de diversifier les instances politiques, d'éviter d'être soumis à la passion d'un seul, et donc de tuer l'absolutisme.

De plus, de la notion d' " air " à celle de «climat », il n'y a qu'un pas, et l'on retrouve en filigrane dans le conte, une discrète référence au livre XVI de L'Esprit des lois, «Des lois dans le rapport qu'elles ont avec la nature du climat». Montesquieu y développe l'idée que les hommes sont différents selon le climat sous lequel ils vivent, et les lois doivent apprécier ces différences. Cette théorie humaniste prend en compte la variété des comportements humains, et met à mal les despotismes, qu'ils soient religieux ou politiques. La publication de ces deux ouvrages est trop proche pour que l'auteur du conte ne pense pas ici à ce développement, alors même qu'elle cite le titre de l'ouvrage de Montesquieu dans le corps du conte ${ }^{26}$. L'auteur ne manque pas d'observer, par le truchement des paroles de Selnozoura - de la même manière que Montesquieu emprunte la voix d'Uzbek dans les Lettres persanes -, que « [l'air] de France par exemple, quoiqu'il arrivât d'ordinaire en plus petite quantité, à cause qu'il s'évapore facilement, ne laissait pas de se faire remarquer, surtout lorsqu'il avait été pris dans la capitale ${ }^{27}$ ». Elle condamne ainsi le règne des modes et les insuffisances de la tête de son propre pays.

38 La diversité des points de vue, la prise en compte de la variété des hommes et de leurs coutumes, ainsi que de leurs lois, permet de rendre raisonnable la tête d'un pays. Car s'il s'agit ordinairement de confronter les pouvoirs, les échanges se font bien souvent par armées interposées. Ainsi dans l'Histoire secrète du prince Croqu'Etron et de la princesse Foirette $^{28}$, toute allégorie scatologique détournée, il est bel et bien question de la chose militaire. Mlle de Lubert fait reposer le pouvoir d'une nation sur la puissance de son armée, car, en effet, les interactions entre les royaumes se font bien souvent au travers de rapports de forces. Là, l'auteur semble prôner une politique antimilitariste, qui mettrait en avant les échanges des idées plutôt que des coups de feu. On voit se dessiner dans ce conte l'éloge de la diplomatie, qui remplacerait, dans un contexte idéal, la guerre, fléau des nations et témoin d'un impérialisme mortifère. Sans vouloir révéler le dénouement, la mort du héros - peu commune dans les contes merveilleux, proclame la défaite d'une politique de la violence.

Ces quelques exemples mettent en valeur l'ouverture d'un pouvoir sur des cultures diverses, sur l'observation de gouvernements différents. Cependant, pour aider les monarques, Mlle de Lubert fait appel aux philosophes, et suivant l'exemple de Voltaire qui se lie avec les dirigeants européens friands de ses conseils, elle fait de ses contes un véritable éloge de la monarchie éclairée.

La féerie permet aussi une autre manière d'approcher la politique, celle que procure l'utopie. "Le Siècle des Lumières [...] est l'âge d'or de l'utopie au sens de la représentation littéraire de sociétés idéales fictives. En prenant le mot au sens large, on peut aller jusqu'à affirmer que les Lumières sont une utopie » : c'est ainsi que Heinrich Hudde ouvre l'article «Utopie " du Dictionnaire européen des Lumières ${ }^{29}$. Dans un siècle où L'Utopie de Thomas More est réédité en 1643, 1715, 1730, 1741 et 1780 -, l'utopisme propose souvent un modèle de législation dont le postulat est de «vivre selon la nature ${ }^{30} »$. 
41 Tecserion, publié en 1737, ouvre même l'utopie à l'univers interplanétaire, deux ans avant Micromégas, de Voltaire. L'auteur propose de projeter prince et princesse, personnages politiques en devenir, sur la Planète de Vénus : utopie de l'Arcadie, en un sens, qui présente une idylle champêtre en peuplant cette planète de bergers et de bergères ; utopie amoureuse et découverte des corps et de la sensualité également, mais aussi, et surtout, utopie politique :

[...] le soleil qui semblerait devoir rendre cette planète inhabitable par sa chaleur, absorbe ses rayons dans une vaste atmosphère qui entoure notre air supérieur, ainsi nous ne sentons qu'une chaleur tempérée et même toujours égale, car nous avons un printemps continuel, et celui qui règne chez vous n'est qu'une influence de notre globe, puisqu'alors les habitants de la Terre goûtent à peu près notre bonheur.

La nuit ne nous enveloppe point aussi d'épaisses ténèbres, parce qu'elle est presque toute partagée entre les crépuscules et les aurores, qui nous tiennent lieu de lune ; nous en tirons aussi de grands avantages: souvent une rigoureuse beauté après avoir désespéré son amant pendant la clarté du jour, devient plus traitable dans l'obscurité séduisante de la nuit ; [...] la loi fondamentale de ce pays, est d'aimer, et on l'observe exactement en changeant toujours d'objet, puisqu'en rompant un engagement pour un autre, on ne cesse point d'aimer. [...]

L'Amour, [...] ce Dieu tout puissant, cette souveraine intelligence que nous servons avec un culte si pur, est l'inventeur de notre langue; quoiqu'elle ne soit pas régulière, nous nous faisons comprendre sans peine, et nous persuadons sans éloquence. [...] Nous parlons donc le langage des Dieux: c'est une langue universelle, car tout mortel peut l'entendre, et le plus amoureux est celui qui la parle le mieux. Notre Académie composée des amants les plus spirituels, ne s'occupe point à l'enrichir ni à la reformer, elle respecte l'usage, s'applique à le conserver, et tient pour assuré que le beau langage est celui qui est reçu généralement: Cet auguste tribunal administre aussi la justice, et juge les coupables. Ici la forme n'emporte jamais le fond, car nous détestons la chicane et ses formalités; nos juges, quoique jeunes, ont des lumières et de l'intégrité ; d'ailleurs nos lois sont si simples, et en si petit nombre, qu'il suffit de les consulter pour prononcer sur tous les cas. Nous ne connaissons guère d'autres crimes que la jalousie, la froideur, l'indifférence, l'infidélité et la coquetterie. [...]

Vous saurez encore [...] que nous sommes gouvernés par un Sacrificateur, qui est roi en même temps; son autorité est de dix années, et il partage l'honneur de la sacrificature avec une Bergère [...]. Ils sont élus l'un et l'autre dans une assemblée générale ; on a surtout égard à leur beauté et à leur tendresse ; car quoiqu'ils puissent se quitter, on examine au moins si jusqu'alors ils se sont aimés de bonne foi ${ }^{31}$.

Cet endroit imaginaire, aux confins du système solaire, où est projeté la princesse Belzamine, propose en terme d'organisation sociale des idées nouvelles et propres aux volontés de réformes des Lumières. Cette société à certains égards paraît proche de la nôtre, ce qui rend ces nouvelles idées probables.

Comme la société française, le monde de la Planète de Vénus possède un dieu, qui en l'occurrence ici est une figure féminine (Vénus). Comme la France au XvIII siècle, elle possède un roi, garant du droit divin (le sacrificateur). Dans ce "nouveau monde", cependant, règne l'égalité entre les hommes et les femmes, tant par leurs attributs et leurs qualités que dans le partage du pouvoir. Effectivement, on note ici que l'accès des femmes au pouvoir est naturel, et non pas occasionnel - comme lors de périodes de régence.

44 La société proposée est, de plus, pacifique de par l'inspiration commune qui motive les autochtones à l'amour, objectif universel. Sur la planète de Vénus, le printemps est 
éternel et marque la pérennité de l'éveil des sens, de la connaissance, de la fertilité, mais aussi de la tempérance dans le climat ainsi que dans les relations. La nuit n'est jamais complète dans ce coin du conte : l'obscurantisme n'a donc pas de développement possible, et c'est une lumière douce qui règne pour atténuer en chacun tout soupçon de laideur. Ainsi la beauté réside en toute chose.

La population de la Planète de Vénus dispose d'un langage universel, dont l'évolution est naturelle : les paroles sont simples, les lois sont donc simplement ordonnées, ce qui permet, l'égalité de tous dans la compréhension des textes juridiques. Ce langage est "le langage des dieux ", qui donne libre accès à la connaissance absolue, celle que la France catholique a perdu par la faute d'Ève. Ce langage est observé par l'« Académie »: on peut voir ici la critique d'une autre institution tyrannique du langage et des Lettres, qui dirige la langue française, fige l'esprit de main de maître et se targue d'une érudition élitiste. L'Académie présente sur la planète de Vénus se porte garante au contraire de l'accessibilité de la langue, et donc des idées, ainsi que du naturel de son évolution.

Dans cette contrée idéale, jeunesse est synonyme de sagesse, et le corps des magistrats en est témoin. Le sacrificateur, de plus, est choisi parmi plusieurs candidats issus du peuple ; il est élu selon un suffrage et pour une durée déterminée. Le pouvoir est de fait accordé au peuple, selon une nouvelle idée de démocratie.

Les utopies, chez Mlle de Lubert, proposent une politique nouvelle, loin du pouvoir absolu dispensé par un seul homme-dieu. Le pouvoir ne doit pas émaner d'un seul " soleil », d'un monarque absolu et mégalomane, mais doit prendre en compte les voix d'un peuple multiple. Ces voix doivent être dirigées vers la sagesse politique par la lumière des philosophes.

"Les fées que la philosophie doit toujours conduire ${ }^{32}$ " sont les figures, mélanges d'humain et de divin, choisies par Mlle de Lubert pour prononcer de justes critiques sur ces rigueurs trop grandes, que les Lumières du siècle doivent travailler à abolir.

Les règles précises du conte de fées permettent à l'auteur une liberté que l'on ne trouverait pas dans une liberté formelle, où l'auteur serait trop attendu. La ruse consiste à entraîner le lecteur à prendre possession d'un sujet innocent, pour, en cours de route, en faire un sujet politique. C'est ainsi que Mlle de Lubert, sous couvert de " faire des riens ${ }^{33}$ " appelle le lecteur à s'imprégner de la chose politique. L'auteur se sert de la liberté que lui procure les contraintes du genre de la féerie pour dénoncer les travers de la société du XVIII ${ }^{e}$ siècle.

C'est par le biais de la fantaisie que Mlle de Lubert discute sérieusement des ridicules des tyrans, de la vanité de la cour, et va jusqu'à prédire la chute de la cité, de la polis à proprement parler. Après avoir présenté un état de fait, elle exige de ses contes qu'ils déploient les idéaux politiques de l'esprit des Lumières. C'est à des esprits candides qu'elle va donner la parole: involontaires sujets de l'étonnement philosophique, ils s'ouvriront au monde politique. Le nécessaire comportement cosmopolite de ces princes permet au lecteur d'être attentif aux expériences de l'étranger, tant par l'observation distante d'autres politiques, que par l'immersion dans l'utopie la plus développée. Le premier homme de l'État, ainsi pénétré d'influences multiples prouve par là même les bienfaits d'une politique éclairée. Mlle de Lubert érige le conte de fées à la hauteur d'un traité politique. 


\section{NOTES}

1. Manuel, lexique ou dictionnaire des mots français dont la signification n'est pas familière à tout le monde, par l'Abbé Prévost, Paris, Didot, 1750. Article « Fée ».

2. Dans la première édition de Tecserion, en 1737, « Il y a de la raison à s'amuser par la folie » tient lieu de sous-titre au conte, cependant que dans les éditions suivantes, l'éditeur ou l'auteur a choisi de le faire figurer comme titre du Discours préliminaire. Tecserion, Il y a de la raison à s'amuser par la folie, à Paris, chez Prault père, Quai de Gèvres, au Paradis, 1737.

3. Tecserion, ouvr. cité, Discours préliminaire (non paginé).

4. Idem.

5. Le Robert, Dictionnaire historique de la langue française, sous la direction d'Alain Rey, Dictionnaires Le Robert, Paris, 2000.

6. Tecserion, ouvr. cité, Discours préliminaire (non paginé).

7. En italiques dans le texte. La Princesse Sensible et le prince Typhon, conte, à La Haye, 1743 , p. 8.

8. Tecserion, ouvr. cité, p. 30-31.

9. Blancherose, conte, à Londres, 1751, p. 8.

10. La Princesse Coqued'oeuf et le prince Bonbon, Histoire aussi ancienne que véritable, traduite de l'Arabe par M.L. Degbacobub, à La Haye, chez J. Neaulme, 1745, p. 1-2.

11. La Princesse Lionnette et le prince Coquerico, ouvr. cité, p. 1-2.

12. La Princesse Sensible et le prince Typhon, ouvr. cité, p. 14.

13. Ibid. p. 17.

14. Ibid. p. 18.

15. Ibid. p. 18

16. Ibid. p. 11.

17. Cornichon et Toupette, Histoire fée, à La Haye, chez Pierre de Hondt, 1752, p. 27-28.

18. Voir Mme d'Aulnoy, La Biche au bois, dans Contes des fées, suivis de Contes nouveaux ou Les Fées à la mode, Paris, Champion, 2004.

19. Voir l'éducation de la princesse Belzamine dans Tecserion, Mlle de Lubert.

20. Cornichon et Toupette, ouvr. cité, p. 32-33.

21. La Princesse Lionnette et le prince Coquerico, ouvr. cité, p. 78.

22. Ibid. p. 82.

23. Dans Cornichon et Toupette, ouvr. cité, p. 70-71: «Je ne puis m'empêcher de faire ici une réflexion qui n'aurait pas échappé à mon auteur s'il eût vécu de nos jours [...]. C'est que cet endroit est si conforme pour le fonds, à l'opinion établie dans quelques chapitres du livre de l'Esprit des lois, qu'on pourrait soupçonner son auteur de plagiat, si on le croyait versé dans l'histoire des fées, mais il y a peu d'apparence ».

24. Tecserion, ouvr. cité, p. 137-140.

25. Cornichon et Toupette, ouvr. cité, p. 67-70.

26. Ibid.

27. Ibid. p. 65-66.

28. Pour la question de l'attribution de ce conte à Mlle de Lubert, voir la notice d'Aurélie Zygel-Basso, dans Mademoiselle de Lubert, Contes, Paris, Champion «Bibliothèque des génies et des fées, 14 », 2005, p. 551-552. 
29. Dictionnaire européen des Lumières, publié sous la direction de Michel Delon, Paris, PUF, 1997.

30. Thomas More, L'Utopie, (Thierry Martin, Louvain, 1516), Librio, 2001 (p. 79) : Note de Marcelle Bottigelli : «Cette morale des Utopiens participe à la fois de la morale d'Épicure et de celle des Stoïciens. «Vivre selon la nature » est une formule stoïcienne, qui s'identifie à « vivre selon la raison » puisque pour les philosophes, la nature est pénétrée de raison, c'est-à-dire est conforme à des lois définies que le sage peut connaître, et dans la contemplation desquelles il trouve la béatitude. »

31. Tecserion, ouvr. cité, p. 176-186.

32. La Princesse Sensible et le prince Typhon, ouvr. cité, p. 67.

33. Tecserion, ouvr. cité, Discours préliminaire.

\section{RÉSUMÉS}

«Faire durer une chose sans arriver au fait, afin de faire perdre de vue le but de l'entreprise » est une des définitions que prend le terme «amuser» à la fin du XVII siècle. En considérant l'amusement comme maître mot de son œuvre, Mlle de Lubert va tromper l'esprit du lecteur. Pris au piège de la légèreté du genre, il est contraint, sans s'en apercevoir, de renouveler sa réflexion politique. Rois poltrons ou dirigeants tyranniques vont être détrônés par le ridicule et le monstrueux que soutient la féerie. Sous la satire merveilleuse, la cour devient un microcosme mortifère, et la critique s'étend même jusqu'au sein des salons précieux. L'auteur nous demande maintes fois d'observer l'Histoire pour en retenir la leçon. Elle provoque chez ses personnages des attitudes philosophiques d'inspiration cosmopolite : l'un va devoir effectuer un Grand Tour, l'une assiste à un débat sur la notion d'étranger, une autre encore se rend compte de la nécessité de séparer les pouvoirs. Forte des leçons de politique qu'elle transmet par ses contes, Mlle de Lubert rend la féerie utile à la philosophie. Qui se méfie d'un conte de fées ? On y peut tout dire. Alors, quand l'utopie redouble la force du conte, la satire religieuse approfondit la leçon politique. L'indifférence sexuelle prend ainsi des airs de scepticisme théologique. Une esthétique du bizarre vient perturber une simple lecture des contes de Mlle de Lubert, remettant en question le pouvoir arbitraire d'une religion qui obscurcit les esprits. Car le démantèlement de la monarchie absolue ne se fait pas sans égratigner un Dieu tout puissant et misogyne. Et si la différence entre Dieu et la Fée était simplement une différence de sexe, l'un et l'autre fruits d'une imagination débridée?

Lessons of the Enlightenment by Mlle de Lubert. "To make a thing last without arriving at the fact, in order to make one forget its real object" is one of the definitions of the verb amuser at the end of the $17^{\text {th }}$ century. Taking the amusement as the master word of her fairy work, Mlle de Lubert will mislead the spirit of the reader. He'll fall into the trap of the flightiness of the tale, and will unconsciously revive his political meaning. The fairyhood will dethrone coward kings or despot monarchs giving them ridiculous and monstruous features. Under the satire, the court becomes an empty world, and a vain microcosm, while criticism extends all the way to the salons précieux. The author asks us many times to observe and retain the lessons that History gives. Amongst her characters, we'll note philosophical attitudes to cosmopolitism: one of them will have to carry out a Grand Tour, one attends a discussion about foreigners, another still realizes 
the benefit of the separation of powers. Empowered by the lessons of policy which are transmitted by her tales, Mlle de Lubert makes fairyhood useful for the new philosophy of the Enlightenment. Who'll be suspicious of a fairy tale? There one can say everything! Then, when utopia reinforces the tale, religious satire deepens the political lesson. Thus, sexual indifference takes airs of theological scepticism. An aesthetics of bizarre comes to disturb a simple reading of the tales, calling into question the arbitrary power of religion. Actually the dismantling of the absolute monarchy throws off balance the powerful and misogynous God. And if the difference between God and the Fairy were simply a difference in sex, both being the fruit of an unslung imagination?

\section{AUTEUR}

\section{MARIE VORILHON}

Université Paris 4 Sorbonne 